\title{
Neutron scattering measurements of phonons in nickel at elevated temperatures
}

\author{
M. Kresch, O. Delaire, R. Stevens, J. Y. Y. Lin, and B. Fultz \\ W. M. Keck Laboratory, California Institute of Technology, Mail Stop 138-78, Pasadena, California 91125, USA
}

(Received 7 November 2006; published 9 March 2007)

\begin{abstract}
Measurements of elastic and inelastic neutron scatterings from elemental nickel were made at 10, 300, 575, 875 , and $1275 \mathrm{~K}$. The phonon densities of states (DOSs) were calculated from the inelastic scattering and were fit with Born-von Kármán models of the lattice dynamics. With ancillary data on thermal expansion and elastic moduli, we found a small, negative anharmonic contribution to the phonon entropy at high temperature. We used this to place bounds on the magnetic entropy of nickel. A significant broadening of the phonon DOS at elevated temperatures, another indication of anharmonicity, was also measured and quantified.
\end{abstract}

DOI: 10.1103/PhysRevB.75.104301

PACS number(s): 63.20.Dj, 61.12.Ex, 65.40.Gr

\section{INTRODUCTION}

The free energies of solid phases and their consequences with respect to phase diagrams continues to be an important and productive topic of research. ${ }^{1-11}$ The stabilities of phases at low temperatures (at both ambient and high pressures) can often be understood by determining the enthalpy $H$ with electronic structure calculations in the local-density approximation. At somewhat higher temperatures where entropic contributions to the free energy become important, methods have been available for some time for calculating the configurational entropy of alloys. ${ }^{12-16}$

For pure elements, the entropy $S$ may be written as a sum of contributions from electrons and phonons, $S_{\mathrm{el}}$ and $S_{\mathrm{ph}}$, provided the electrons and phonons do not interact. The entropy of electronic excitations is often calculated from the density of states near the Fermi level, obtained from electronic structure calculations. More recently, frozen phonon or linear response methods used with density-functional calculations have proved effective for calculating the phonon entropy of pure elements and intermetallic compounds. ${ }^{17-20}$ Even for pure elements, the situation becomes more complicated at elevated temperatures, owing to the increasing number of excitations of electrons and phonons and the consequent interactions between them. It is known that vibrational entropy can be altered substantially by electron-phonon or phonon-phonon interactions, but the systematic effects of these interactions on the free energies are not well understood.

The phonon entropy may be subdivided into the contribution from a perfectly harmonic solid $S_{H}$, the contribution due to the dilation of the lattice $S_{D}$, and the remainder, or anharmonic entropy $S_{A}$,

$$
S=S_{\mathrm{el}}+S_{\mathrm{ph}}=S_{\mathrm{el}}+S_{H}+S_{D}+S_{A} .
$$

The sum $S_{H}+S_{D}$ gives a contribution termed "quasiharmonic" entropy. In most cases, the phonons are altered in energy owing to thermal dilation, but the entropy can be assessed with harmonic phonons at elevated temperature. While this approach can provide useful thermodynamic information, it does not account for phonon damping, which is another anharmonic effect of interest at elevated temperature.

From thermodynamics, we calculate $S_{D}$ as

$$
S_{D}=S_{D}(T)=\int_{T_{0}}^{T} \frac{C_{P}-C_{V}}{T^{\prime}} d T^{\prime}=\int_{T_{0}}^{T} \frac{9 K \alpha^{2}}{\rho_{N}} d T^{\prime},
$$

where $C_{P}$ is the heat capacity at constant pressure, $C_{V}$ the heat capacity at constant volume, $K$ the isothermal bulk modulus, $\alpha$ the linear coefficient of thermal expansion, and $\rho_{N}$ the number density-all implicitly functions of temperature. The reference temperature $T_{0}$ is the lowest temperature where experimental data are available. Determination of the anharmonic contribution to the entropy $S_{A}$ requires knowledge of the phonon density of states (DOS) as a function of temperature.

Measurements of the phonon dispersions of bodycentered-cubic (bcc) transition metals ${ }^{21-26}$ have shown that the quasiharmonic model cannot always be reconciled with the experimentally determined entropy of a solid. For chromium, considering only thermal expansion against the bulk modulus severely underestimates the entropy given by the softening of the phonon DOS. ${ }^{21}$ On the other hand, the phonon spectrum of vanadium is largely unchanged over a wide range of temperatures, despite normal thermal expansion. ${ }^{22}$

Wallace $^{3}$ and Eriksson et al. ${ }^{5}$ evaluated the harmonic, quasiharmonic, and anharmonic entropies of transition metals. In the case of nickel, they divide the electronic entropy into a contribution from the noninteracting electrons $S_{E}$ and a contribution from magnetism $S_{M}$ such that

$$
S_{\mathrm{el}}=S_{E}+S_{M}
$$

They determined $S_{E}$ through an ab initio electronic structure calculation, and used it to make an estimate of the sum of the anharmonic phonon and the magnetic entropies,

$$
S_{A}+S_{M}=S-S_{H}-S_{D}-S_{E}
$$

They were, however, unable to separate the two terms on the left-hand side of this equation. Meschter et al. have performed a similar analysis of the heat capacity of nickel, also estimating the anharmonic contributions to the entropy. ${ }^{6}$

By measuring the phonon DOS at high temperatures, we are able to determine precisely the anharmonic contribution to the total entropy of nickel. Previous measurements of the phonon dispersions in nickel were reported by Birgeneau et al.,${ }^{27}$ Hautecler and Van Dingenen, ${ }^{28}$ and deWit and Brockhouse, but all these studies were confined to temperatures 
between 296 and $676 \mathrm{~K}$. The purpose of deWit and Brockhouse's measurements was not specifically to investigate phonon thermodynamics, but rather to look for changes in the phonon modes as the metal went through the Curie transition at $T_{C}=631 \mathrm{~K}$. They reported little change in the phonons through the magnetic transition and in the shifts in the phonon energies that were largely consistent with a quasiharmonic model. They also reported significant broadening of the phonon peaks with increasing temperature, which they suggested may be due to interactions of magnetism with the lattice..$^{29}$

Zoli et al. investigated the broadening of phonons in facecentered-cubic (fcc) noble metals and aluminum. ${ }^{30}$ Using force constants from Born-von Kármán fits to neutron data and third-order elastic constants, they calculated the full width at half maximum $2 \Gamma$ of the phonon peaks. For aluminum and the noble metals, broadening of the phonon peaks is of course not caused by magnetism but by phonon-phonon interactions that shorten phonon lifetimes.

In the present research, we measured the inelastic scattering of neutrons from elemental nickel from 10 to $1275 \mathrm{~K}$, which is about $75 \%$ of the melting temperature. We explain the data analysis and computation involved in extracting a phonon DOS from the scattering, including a technique for separating single phonon scattering from multiphonon and multiple scattering, given in the Appendix. The phonon DOS curves are used to calculate the anharmonic contribution to the entropy, and this is used to bound the value of the magnetic entropy at high temperature. Finally, we discuss the large broadening of phonon peaks at elevated temperature.

\section{EXPERIMENT}

\section{A. Sample preparation}

Ingots of $99.98 \%$ pure nickel were cold rolled to a thickness of $0.45 \mathrm{~mm}$. At this thickness, $10 \%$ of the incident neutrons are scattered by the sample. The cold-rolled pieces were then cut into strips and annealed at $1075 \mathrm{~K}$ in evacuated quartz tubes for $16 \mathrm{~h}$ to relieve stress and induce recrystallization. There were no signs of oxidation on the annealed strips.

\section{B. Neutron scattering measurements}

Inelastic neutron scattering measurements were performed with the Pharos time-of-flight direct-geometry chopper spectrometer at the Los Alamos Neutron Science Center at temperatures of $10,300,575,875$, and $1275 \mathrm{~K}$. For the 10 and $300 \mathrm{~K}$ measurements, the strips of nickel were laid flat in a thin-walled aluminum pan, which was then mounted on a closed-cycle refrigerator. For higher temperatures, a niobium pan was used, and the sample was mounted in a vacuum furnace built by A. S. Scientific. Several thermocouples were used to monitor the temperature of the sample, and it is estimated that the temperature deviations in the sample were no more than $5 \mathrm{~K}$. Measurements of the empty sample pans were also performed at all temperatures.

Details about the Pharos spectrometer at Los Alamos have been given previously. ${ }^{31,32}$ Data were collected for a mini-

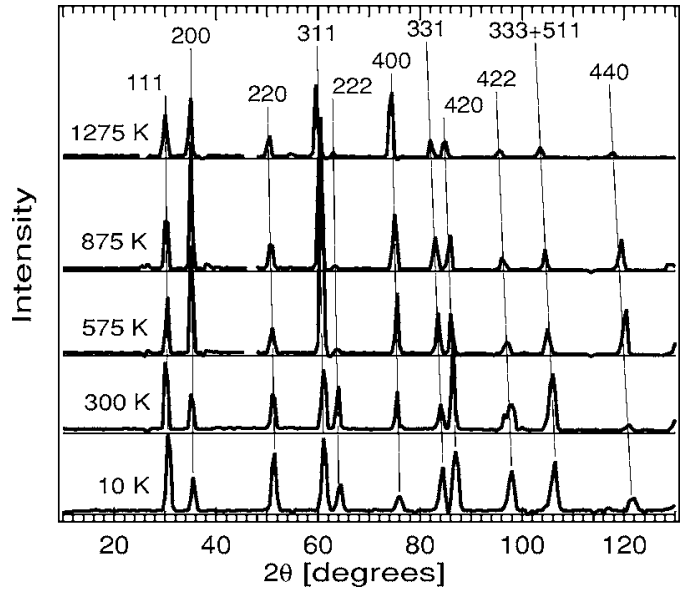

FIG. 1. Diffraction patterns from nickel, taken in situ at temperatures as labeled. The quality of the patterns taken above room temperature is reduced due to the increased background of the furnace.

mum of $4 \mathrm{~h}$ at each temperature, giving on the order of 1 $\times 10^{6}$ counts. The incident energies calculated from the data ranged from 69.3 to $69.6 \mathrm{meV}$. The experimentally determined resolution of the instrument (full width at half maximum) was approximately $2.5 \mathrm{meV}$ at the elastic line and $1.0 \mathrm{meV}$ at the high-energy cutoff of the phonon DOS $(\sim 40 \mathrm{meV})$.

\section{DATA ANALYSIS AND COMPUTATION}

\section{A. General data reduction}

The measured spectra, in time-of-flight, detector number, and pixel were first corrected for the efficiencies of the detectors. This was done using a room-temperature measurement of pure vanadium (a fully incoherent scatterer) for calibration. Next, the background independent of the time-offlight was estimated (as an average over a region in time-offlight having no appreciable scattering from the sample or the environment) and subtracted. The corrected data were normalized by the integrated proton current and converted to intensity $I(2 \theta, E)$ by rebinning into scattering angles, $2 \theta$, ranging from $5^{\circ}$ to $145^{\circ}$ with a bin width of $0.5^{\circ}$ and energy transfers $E$ from -65 to $65 \mathrm{meV}$ with a bin width of $0.5 \mathrm{meV}$. The scattering from the empty pans was subtracted from the data, scaled by $90 \%$ to account for the selfshielding of the sample.

\section{B. Elastic scattering: In situ neutron diffraction}

The scattering with energy transfers between -2.5 and $2.5 \mathrm{meV}$ was used to obtain diffraction patterns from nickel, as shown in Fig. 1. Using Nelson-Riley plots, ${ }^{33}$ the lattice parameter $a$ was found at all measured temperatures. These are listed in Table I and agree with values of the lattice parameter calculated using the accepted temperaturedependent linear coefficient of thermal expansion ${ }^{34}$ and room temperature-lattice parameter. ${ }^{35}$

In addition to those shown in Fig. 1, diffraction patterns were obtained without the furnace at incident neutron ener- 
TABLE I. Experimentally determined lattice parameter $a$ of nickel and shifts of the nickel phonon energies as a function of temperature. Fits using a damped oscillator function and Eq. (6) yield $\Delta$, and $\left\langle E_{T}\right\rangle /\left\langle E_{10}\right\rangle$ was calculated with Eq. (8).

\begin{tabular}{cccc}
\hline \hline$T$ & $a \pm 0.005$ & & \\
$(\mathrm{~K})$ & $(\AA)$ & $\Delta$ & $\left\langle E_{T}\right\rangle /\left\langle E_{10}\right\rangle$ \\
\hline 10 & 3.513 & 1.000 & 1.000 \\
300 & 3.521 & 0.988 & 0.985 \\
575 & 3.540 & 0.970 & 0.963 \\
875 & 3.559 & 0.947 & 0.945 \\
1275 & 3.585 & 0.920 & 0.913 \\
\hline \hline
\end{tabular}

gies of 30,50 , and $70 \mathrm{meV}$, with the 222 peak at $108^{\circ}, 78^{\circ}$, and $64^{\circ}$ in $2 \theta$, respectively. The ratios of peak intensities remained largely unchanged, showing that the sample did not have substantial crystallographic texture. Regardless, sample texture should not affect lattice parameters, as determined with Nelson-Riley plots, and effects of the texture are addressed in Sec. III E and in the Appendix.

\section{Inelastic scattering: $S(Q, E)$ and the density of states}

The data were rebinned again to obtain the intensity $I(Q, E)$, with momentum transfer $Q$ ranging from 0.0 to $13.5 \AA^{-1}$ with a bin width of $0.0675 \AA^{-1}$. Since nickel is ferromagnetic up to the Curie temperature, we excluded scattering at lower momentum transfers, where magnetic scattering is present, to ensure that the scattering from the phonons was dominant. The elastic peak was removed below $\sim 5 \mathrm{meV}$ and replaced with a straight line, corresponding to the continuum limit at low energies. The data were then corrected for multiple and multiphonon scatterings simultaneously, as described in the Appendix. The resulting DOS curves at all temperatures are shown in Fig. 2.

\section{Phonon shifts and broadening}

We expect the broadening of the phonons to take the form of a damped harmonic oscillator function ${ }^{24-26} B\left(\mathcal{Q}, E^{\prime}, E\right)$,

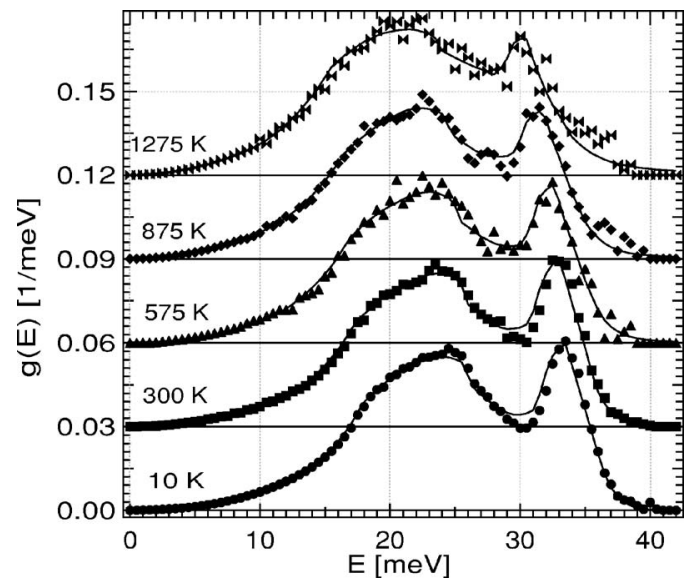

FIG. 2. Phonon DOS for nickel at temperatures indicated. Markers are experimental data, and lines are Born-von Kármán fits. The increase in phonon lifetime broadening and the shifting of modes to lower energies with increasing temperature is evident. The DOSs are offset by integer multiples of $0.03 \mathrm{meV}^{-1}$.

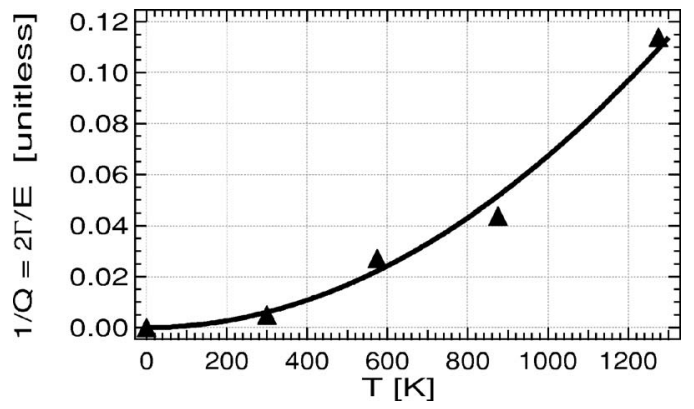

FIG. 3. Triangles show the inverse of the quality factor, $1 / \mathcal{Q}$, as a function of temperature for nickel phonons.

with central energy $E^{\prime}$ and width proportional to the phonon energy $E{ }^{22}$

$$
B\left(\mathcal{Q}, E^{\prime}, E\right)=\frac{1}{\pi \mathcal{Q} E^{\prime}} \frac{1}{\left(\frac{E^{\prime}}{E}-\frac{E}{E^{\prime}}\right)^{2}+\frac{1}{\mathcal{Q}^{2}}} .
$$

The quality factors $\mathcal{Q}$ were determined by a least-squares fit. First, the energies of the $10 \mathrm{~K}$ DOS were scaled by a factor $\Delta$. The rescaled DOS was then convolved with a damped oscillator function to get a candidate fit to the hightemperature DOS.

$$
g_{T}(E) \approx B\left(\mathcal{Q}, E^{\prime}, E\right) * g_{10}\left(E^{\prime} \Delta\right)
$$

where $g_{10}$ is the phonon DOS at $10 \mathrm{~K}$, and the $*$ denotes convolution. The $\mathcal{Q}$ so determined at all temperatures are shown in Fig. 3, and the fits to the DOS are shown in Fig. 4. Approximately, we find

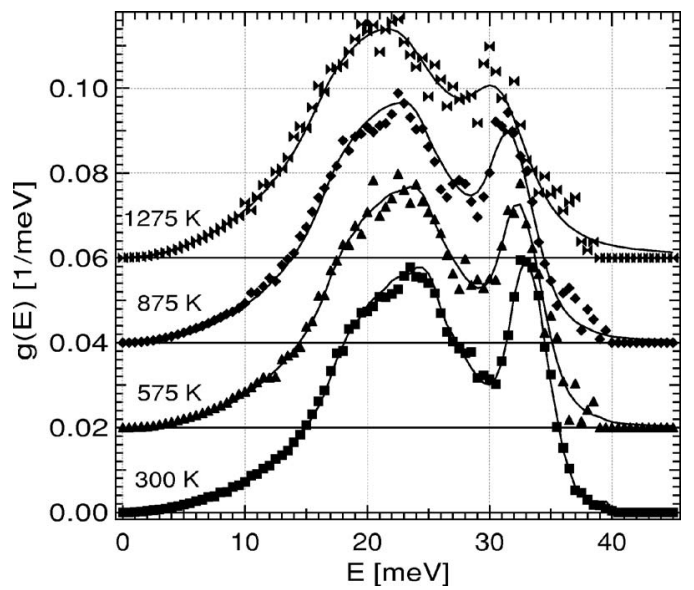

FIG. 4. Phonon DOS of nickel at temperatures as indicated. Markers are experimental data. The lines are fits to the experimental data, acquired by shifting the $10 \mathrm{~K}$ DOS and convolving it with the damped oscillator function, as described by Eqs. (5) and (6). The shifts are listed in Table I. The DOSs are offset by integer multiples of $0.02 \mathrm{meV}^{-1}$. 
TABLE II. Optimized tensor force constants in $\mathrm{N} / \mathrm{m}$ as a function of temperature for fcc nickel. A Cartesian basis is used, where $\langle x y z\rangle$ is the bond vector for the given tensor components.

\begin{tabular}{lcccccc}
\hline \hline & $\langle x y z\rangle$ & $10 \mathrm{~K}$ & $300 \mathrm{~K}$ & $575 \mathrm{~K}$ & $875 \mathrm{~K}$ & $1275 \mathrm{~K}$ \\
\hline$\Phi_{1 x x}$ & $\langle 110\rangle$ & 17.584 & 17.545 & 16.584 & 15.910 & 13.975 \\
$\Phi_{1 x y}$ & & 18.976 & 18.253 & 18.822 & 17.670 & 16.915 \\
$\Phi_{1 z z}$ & & -0.391 & -0.274 & -0.384 & -0.316 & -0.345 \\
$\Phi_{2 x x}$ & $\langle 200\rangle$ & 0.975 & 0.885 & 1.235 & 0.920 & 1.009 \\
$\Phi_{2 y y}$ & & -0.610 & -0.993 & -0.551 & -0.559 & -0.644 \\
$\Phi_{3 x x}$ & $\langle 211\rangle$ & 0.593 & 0.442 & 0.518 & 0.440 & 0.850 \\
$\Phi_{3 x y}$ & & 0.378 & 0.340 & 0.368 & 0.441 & 0.357 \\
$\Phi_{3 y y}$ & & 0.302 & 0.133 & 0.220 & 0.157 & 0.325 \\
$\Phi_{3 y z}$ & & -0.120 & -0.128 & -0.105 & -0.092 & -0.106 \\
$\Phi_{4 x x}$ & $\langle 220\rangle$ & 0.386 & 0.331 & 0.314 & 0.262 & 0.400 \\
$\Phi_{4 x y}$ & & 0.517 & 0.412 & 0.502 & 0.444 & 0.466 \\
$\Phi_{4 z z}$ & & -0.218 & -0.167 & -0.127 & -0.153 & -0.217 \\
$\Phi_{5 x x}$ & $\langle 310\rangle$ & -0.085 & -0.065 & -0.093 & -0.078 & -0.092 \\
$\Phi_{5 x y}$ & & -0.039 & -0.047 & -0.031 & -0.035 & -0.028 \\
$\Phi_{5 y y}$ & & 0.006 & 0.003 & 0.007 & 0.004 & 0.006 \\
$\Phi_{5 z z}$ & & 0.014 & 0.014 & 0.014 & 0.016 & 0.021 \\
\hline \hline
\end{tabular}

$$
\frac{1}{\mathcal{Q}} \approx \frac{2 \Gamma}{E} \approx 6.730 \times 10^{-9} T^{2},
$$

where $T$ is in kelvins. The shifts $\Delta$ and the ratios of the mean phonon energies,

$$
\frac{\left\langle E_{T}\right\rangle}{\left\langle E_{T_{0}}\right\rangle} \equiv \frac{\int E g_{T}(E) d E}{\int E g_{T_{0}}(E) d E},
$$

are presented in Table I.

\section{E. Born-von Kármán models of lattice dynamics}

The DOS curves were fit with a Born-von Kármán model of the lattice dynamics. ${ }^{36,37}$ Force constants out to fifthnearest neighbors $(5 \mathrm{NN})$ were optimized using a gradient search method. The fits at all temperatures, convolved with the damped oscillator function and a Gaussian instrument resolution function, are shown in Fig. 2. The optimized force constants are listed in Table II.

As a check on our calculated force constants and on the effects of sample texture on our determination of the DOS, comparisons were made to the dispersion curves measured by deWit and Brockhouse. ${ }^{29}$ Our $300 \mathrm{~K}$ model is in good agreement with the results of these authors. Trends in the dispersions with respect to temperature are also in agreement.

\section{RESULTS AND DISCUSSION}

At all temperatures, both the longitudinal and the transverse force constants decrease rapidly with nearest-neighbor distance. (These constants were found by diagonalizing the force constant tensors $\Phi$. The longitudinal force constant was determined by comparison to the projection of $\Phi$ onto the bond vector $\langle x y z\rangle$, and the transverse modes were taken to be the remaining two eigenvalues.) Only the first-nearestneighbor longitudinal force constants show a monotonic decrease with temperature. They are almost solely responsible for the shift of the DOS to lower energies with increasing temperature. All longitudinal force constants are plotted in Fig. 5.

The softening of the measured phonon DOS is consistent with that found by deWit and Brockhouse. They found $\left\langle E_{573}\right\rangle /\left\langle E_{295}\right\rangle=0.976,{ }^{29}$ and we find $\left\langle E_{575}\right\rangle /\left\langle E_{300}\right\rangle=0.978$. To find the entropy from the softening of the DOS, we use the expression for the phonon entropy,

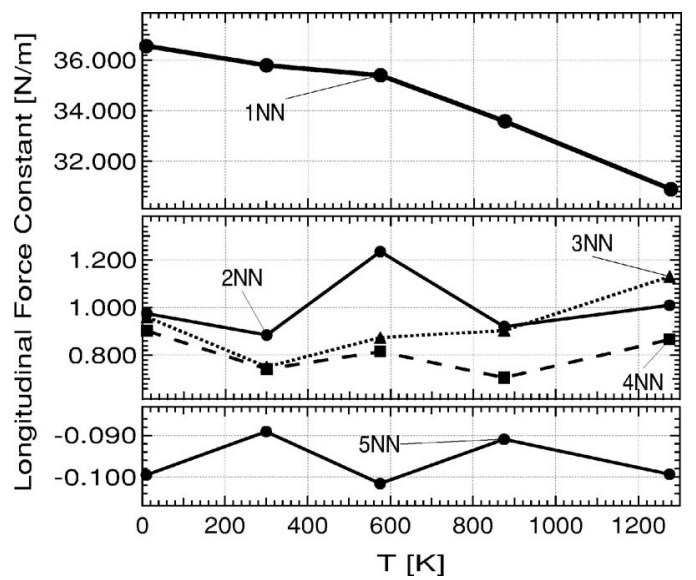

FIG. 5. Longitudinal force constants for first- through fifthnearest neighbors as indicated. 


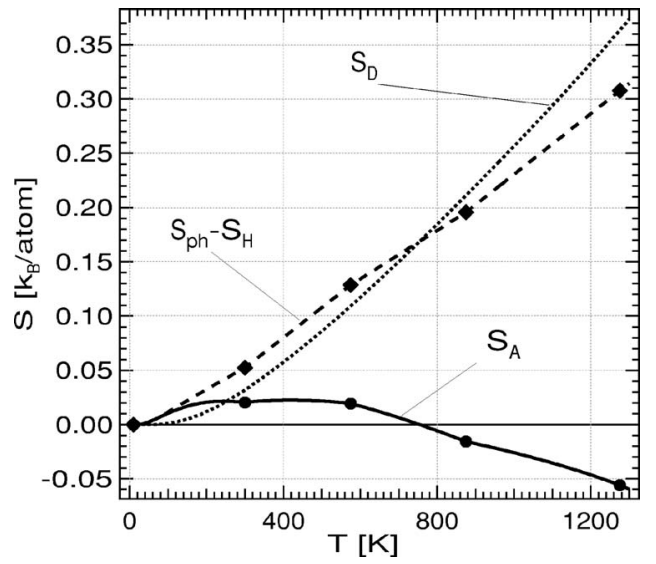

FIG. 6. Entropy of dilation and anharmonic entropy. Markers denote experimental data points-they are connected by linear interpolation of the neighboring DOSs. The dotted line is computed from Eq. (2), the dashed line from Eq. (10), and the solid line is the difference of the other two. The negative entropy signifies that the phonons do not soften as much as one would expect from the expansion of the lattice.

$$
S_{\mathrm{ph}}\left(T, T_{p}\right)=\int_{0}^{\infty} g_{T}\left[\left(n_{T_{p}}+1\right) \ln \left(n_{T_{P}}+1\right)-n_{T_{P}} \ln \left(n_{T_{P}}\right)\right] d E,
$$

where $g_{T}$ is the phonon DOS at temperature $T$ and $n_{T_{P}}$ is the Planck distribution at temperature $T_{P}$ (both $g_{T}$ and $n_{T_{P}}$ are functions of $E$ ). We seek the change in entropy owing to changes in the phonon states, not from changes in phonon occupancy. We calculate the difference between the total phonon entropy and the harmonic phonon entropy as

$$
S_{\mathrm{ph}}-S_{H}=S_{\mathrm{ph}}(T, T)-S_{\mathrm{ph}}\left(T_{0}, T\right) .
$$

We now compare the entropy of phonon softening to the entropy of dilation. With tabulated data for the elastic constants of nickel, ${ }^{38}$ we use Eq. (2), with $T_{0}=10 \mathrm{~K}$, to calculate, $S_{D}$, the entropy of dilation. Figure 6 shows the entropy of dilation and $S_{\mathrm{ph}}-S_{H}$, as determined with Eqs. (9) and (10). The agreement is generally good. The anharmonic entropy, shown as a solid line, is the difference between these two curves. Over the entire temperature range, the anharmonic entropy is bounded by $-0.08<S_{A}<0.05 k_{B}$ /atom, where we have already incorporated our errors of $\pm 0.02 k_{B}$ /atom. Negative values indicate phonons that are slightly stiffer than they would be if their energies were determined by lattice expansion against the bulk modulus alone. This is the case above $700 \mathrm{~K}$, where we have $-0.08<S_{A}<0.03 k_{B}$ /atom. The crossover from positive to negative appears to occur somewhere in the vicinity of the Curie temperature, and the trends in the sign of $S_{\mathrm{ph}}-S_{H}$ appear to be consistent over either the ferromagnetic or the paramagnetic regions.

From Wallace's plots of $S_{\mathrm{el}}$ and $S-S_{\mathrm{ph}},{ }^{3}$ we find the sum of the magnetic and anharmonic contributions to the entropy to be approximately $0.23 k_{B}$ /atom at $1275 \mathrm{~K}$. Wallace assumes that the anharmonic contribution is zero and attributes the entire quantity to magnetic entropy. It appears that the magnetic entropy is slightly larger. At $1275 \mathrm{~K}$, we find $-0.08<S_{A}<-0.04 k_{B}$ /atom; subtracting these values from $S_{A}+S_{M}$, we obtain $0.27<S_{M}<0.31 k_{B}$ /atom. Figure 6 suggests that the anharmonic entropy will decrease linearly with temperature above $1273 \mathrm{~K}$, so the magnetic entropy at melting may be larger than what was previously suggested.

The values of $\mathcal{Q}$ shown in Fig. 3 are related to the full widths at half maximum $2 \Gamma$ of the phonon peaks, through the equation $1 / \mathcal{Q} \approx 2 \Gamma / E$. Values of a similar magnitude were found experimentally for bcc titanium, zirconium, and hafnium, ${ }^{24-26}$ and our $2 \Gamma$ values are also comparable in magnitude to the values found by Zoli et al. for phonon-phonon interactions in aluminum and the noble fcc metals. ${ }^{30}$ The quadratic form of $1 / \mathcal{Q}$ seen in Fig. 3 is also consistent with phonon-phonon interactions.

Further work calculating the phonon linewidths in the manner of Zoli et al..$^{30}$ might be of interest, and the necessary third-order elastic constants are available for nickel. ${ }^{39,40}$ Also, trends in the entropy indicate that a reinvestigation of the phonon modes of nickel around the Curie temperature may be worthwhile.

\section{CONCLUSION}

Phonon DOS curves of fcc nickel were measured by timeof-flight neutron spectrometry over a wide range of temperatures spanning from 10 to $1275 \mathrm{~K}$. The softening of the DOS was generally consistent with the softening expected from expansion of the lattice against the bulk modulus, but the softening is less than expected at high temperatures. we are able to bound the entropic contribution from phonon anharmonicity to $-0.08<S_{A}<-0.04 K_{B} /$ atom at $1275 \mathrm{~K}$. This, in turn, bounds the contribution of the magnetic entropy to $0.27<S_{M}<S_{M}<0.31 k_{B} /$ atom, at $1275 \mathrm{~K}$. Additionally, we found that there is significant broadening of the phonons with increased temperature, which we tentatively attribute to phonon-phonon interactions.

\section{ACKNOWLEDGMENTS}

We thank Frans Trouw and Markus Hehlen for their support during the Pharos experiment. This work has benefited from the use of the Los Alamos Neutron Science Center at the Los Alamos National Laboratory. This facility is funded by the U.S. Department of Energy under Contract No. DEAC52-06NA25396. This work was supported by the Department of Energy through the Basic Energy Sciences Grant DE-FG02-03ER46055.

\section{APPENDIX: SIMULTANEOUS MULTIPHONON AND MULTIPLE SCATTERING CORRECTIONS}

Corrections for multiple scattering have been performed in many ways, from subtracting a constant from the data ${ }^{41}$ to full Monte Carlo simulations. ${ }^{42}$ At high temperatures, the former does not account for the slope of the scattering past the cutoff energy. The latter can be computationally intensive and requires details of the shape of the sample. Here, we take an approach of intermediate complexity. For both multiple scattering and multiphonon scattering, a two-scattering pro- 


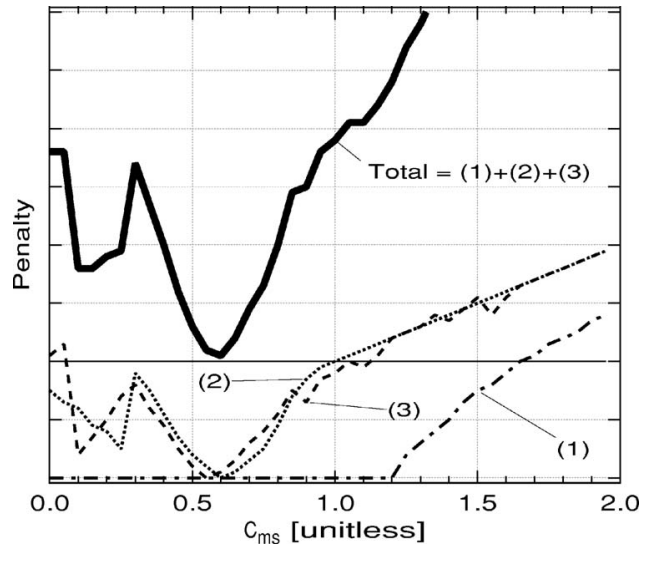

FIG. 7. Penalty functions for nickel at $300 \mathrm{~K}$, as defined in the text. The dash-dotted line (1) relates to the overall fit, the dotted line (2) relates to the noise near the incident energy, and the dashed line (3) relates to the slope near the incident energy. The solid line is the sum of these three contributions (offset).

file involves a convolution of two single-scattering profiles. In either case, the idea is that an $n$-phonon-scattering profile, $P^{n}(E)$ is related to the one-phonon-scattering profile $P^{1}(E)$ through the recursion relation

$$
P^{n}(E)=\int_{-\infty}^{\infty} P^{n-1}\left(E^{\prime}\right) P^{1}\left(E-E^{\prime}\right) d E^{\prime} .
$$

For multiple scattering processes, the $n$-phonon probability function has additional position and momentum dependencies, which do not appear for multiphonon scattering processes. Sears et al. ${ }^{41}$ argue that the integrals for multiple scattering are related to those for the multiphonon scattering through slowly varying functions of $Q$ and $E$. Here, we take these functions to be constants, $a_{n}$. In essence, we make the approximation that the position and momentum dependencies can be factored out. Thus,

$$
I(Q, E)=N^{\prime}\left[\sum_{n=1}^{\infty}\left(1+a_{n}\right) S^{n}(Q, E)\right],
$$

where $I(Q, E)$ is the experimentally determined total scattering (including multiple scattering), $S^{n}(Q, E)$ is the $n$-phonon scattering (both creation and annihilation), and $N^{\prime}$ is a normalization constant. Note that $I(Q, E)$ is distinct from the scattering function $S(Q, E)$, which does not include multiple scattering. ${ }^{43}$ [When we stripped the elastic peak from the data, the dominant multiple elastic scattering is removed, so the index $n$ in Eq. (A2) starts at 1 rather than 0.]

Consistent with this factoring of $Q$ and $E$ dependencies, we make the incoherent approximation, ${ }^{44}$

$$
S_{\mathrm{coh}}^{n}(Q, E)=\frac{\sigma_{\mathrm{coh}}}{\sigma_{\mathrm{inc}}} S_{\mathrm{inc}}^{n}(Q, E),
$$

where we apply this equation to the one-phonon terms as well as all higher orders. The last step in our procedure will be to assess any error this has introduced into our analysis.

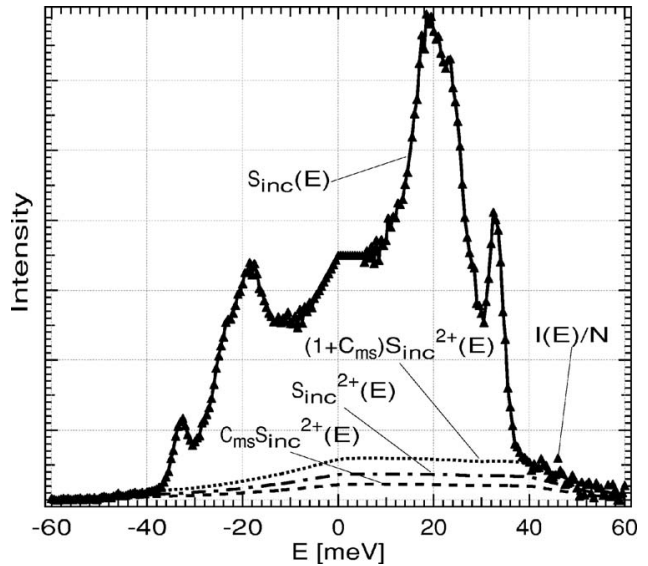

FIG. 8. Best fit to scattering for nickel at $300 \mathrm{~K}$. The triangles are the normalized experimental scattering, $I(E) / N$. The solid line shows the fit, $S_{\text {inc }}^{1}(E)+\left(1+C_{\mathrm{ms}}\right) S_{\text {inc }}^{2+}(E)$. The dashed line is the multiple scattering, $C_{\mathrm{ms}} S_{\text {inc }}^{2+}(E)$. The dash-dotted line is the multiphonon scattering, $S_{\text {inc }}^{2+}(E)$. The dotted line is the sum, $\left(1+C_{\mathrm{ms}}\right) S_{\text {inc }}^{2+}(E)$. The resultant DOS is the $300 \mathrm{~K}$ experimental DOS shown in Figs. 2 and 4 .

Our next assumption is that $a_{n}=C_{\mathrm{ms}}^{\prime}$ for all $n \geqslant 2$, where $C_{\mathrm{ms}}^{\prime}$ is a single constant that relates the multiple scattering to the multiphonon scattering. Since the multiphonon scattering drops off rapidly with increasing $n$, this approximation will only have a small effect on our results. The final normalization is performed with the total scattering, so the factor 1 $+a_{1}$ is included in the normalization constant. We find

$$
I(Q, E)=N\left[S_{\text {inc }}^{1}(Q, E)+\left(1+C_{\mathrm{ms}}\right) S_{\text {inc }}^{2+}(Q, E)\right],
$$

where $N=N^{\prime}\left(1+a_{1}\right)\left(1+\sigma_{\text {coh }} / \sigma_{\text {inc }}\right)$ is the normalization constant and $1+C_{\mathrm{ms}} \equiv\left(1+C_{\mathrm{ms}}^{\prime}\right) /\left(1+a_{1}\right)$. Also, for notational convenience,

$$
S^{j+}(Q, E) \equiv \sum_{n=j}^{\infty} S^{n}(Q, E) .
$$

For a cubic crystal and a fixed value of $C_{\mathrm{ms}}$, we can now find the DOS by solving Eq. (A4) in the manner described by Sears et al. ${ }^{41}$

Since we do not know the value of $C_{\mathrm{ms}}$ a priori, we generate a list of possible values and solve for the DOS at each one. In the current study, values of $C_{\mathrm{ms}}$ between 0.0 and 2.0 were tested. What remains then is to select the "best" DOS from those generated with the different $C_{\mathrm{ms}}$. This was done by minimizing a penalty function constructed to find the DOS that produced $S(E)$ that best satisfied the following conditions:

$$
\frac{I(E)}{N}=S_{\mathrm{inc}} 1(E)+\left(1+C_{\mathrm{ms}}\right) S_{\mathrm{inc}}^{2+}(E),
$$

where the implied sum over Q allows us to compare the partially coherent scattering on the left with the totally incoherent scattering on the right. 
(2) The experimental noise at energy transfers near the incident energy oscillates about $\left(1+C_{\mathrm{ms}}\right) S_{\mathrm{inc}}^{2+}(E)$.

(3) At energy transfers near the incident energy, the slope of a linear fit to the experimental noise matches the slope of a linear fit to $\left(1+C_{\mathrm{ms}}\right) S_{\mathrm{inc}}^{2+}(E)$.

These three criteria are correlated, but are not identical. For nickel at $300 \mathrm{~K}$, these three contributions and their sum are shown in Fig. 7. Figure 8 shows the best fit to the normalized scattering, $I(E) / N$ for nickel at $300 \mathrm{~K}$, which had $C_{\mathrm{ms}}=0.6$.
The DOS curves obtained this way were fit with a Bornvon Kármán model, from which all phonon contributions to the scattering, both coherent and incoherent, were calculated. With these results, and with the final value for $C_{\mathrm{ms}}$, the calculation was checked against the measured scattering, which showed that the effects of assuming all the scattering to be incoherent were negligible. This implies that the effects of sample texture were negligible as well. It was our experience that this procedure worked well for the present case of nickel, and also worked for cases of other bcc and fcc materials.
${ }^{1}$ N. Bock, D. C. Wallace, and D. Coffey, Phys. Rev. B 73, 075114 (2006).

${ }^{2}$ N. Bock, D. Coffey, and D. C. Wallace, Phys. Rev. B 72, 155120 (2005).

${ }^{3}$ D. C. Wallace, Statistical Physics of Crystals and Liquids: A Guide to Highly Accurate Equations of State (World Scientific, Singapore, 2002), Chap. 19, pp. 202-203.

${ }^{4}$ D. C. Wallace, Phys. Rev. E 56, 1981 (1997).

${ }^{5}$ O. Eriksson, J. M. Wills, and D. Wallace, Phys. Rev. B 46, 5221 (1992).

${ }^{6}$ P. J. Meschter, J. W. Wright, C. R. Brooks, and T. G. Kollie, J. Phys. Chem. Solids 42, 861 (1981).

${ }^{7}$ P. B. Allen and J. C. K. Hui, Z. Phys. B 37, 33 (1980).

${ }^{8}$ M. E. Manley, R. J. McQueeney, B. Fultz, T. Swan-Wood, O. Delaire, E. A. Goremychkin, J. C. Cooley, W. L. Hults, J. C. Lashley, R. Osborn, and J. L. Smith, Phys. Rev. B 67, 014103 (2003).

${ }^{9}$ M. E. Manley, R. J. McQueeney, B. Fultz, R. Osborn, G. H. Kwei, and P. D. Bogdanoff, Phys. Rev. B 65, 144111 (2002).

${ }^{10}$ R. Stedman, L. Almqvist, and G. Nilsson, Phys. Rev. 162, 549 (1967)

${ }^{11}$ G. Grimvall, J. Häglund, and A. Fernandez Guillermet, Phys. Rev. B 47, 15338 (1993).

${ }^{12}$ R. Kikuchi, Phys. Rev. 81, 898 (1951).

${ }^{13}$ J. M. Sanchez, F. Ducastelle, and D. Gratias, Physica A 128, 334 (1984).

${ }^{14}$ D. de Fontaine, in Solid State Physics, edited by H. Ehrenreich, F. Seitz, and D. Turnbull (Academic, New York, 1979), Vol. 34.

${ }^{15}$ F. Ducastelle, Order and Phase Stability in Alloys (NorthHolland, Amsterdam, 1991), Chap. 4.

${ }^{16}$ A. Zunger, in Statics and Dynamics of Alloy Phases, edited by P. E. A. Turchi and A. Gonis (Plenum, New York, 1994), p. 361.

${ }^{17}$ Y. Y. Ye, Y. Chen, K. M. Ho, B. N. Harmon, and P. A. Lindgard, Phys. Rev. Lett. 58, 1769 (1987).

${ }^{18}$ V. Ozolins, C. Wolverton, and A. Zunger, Phys. Rev. B 58, R5897 (1998).

${ }^{19}$ V. Ozolins and M. Asta, Phys. Rev. Lett. 86, 448 (2001).

${ }^{20}$ A. van de Walle and G. Ceder, Rev. Mod. Phys. 74, 11 (2002).

${ }^{21}$ J. Trampenau, W. Petry, and C. Herzig, Phys. Rev. B 47, 3132 (1993).

${ }^{22}$ P. D. Bogdanoff, B. Fultz, J. L. Robertson, and L. Crow, Phys. Rev. B 65, 014303 (2001).

${ }^{23}$ F. Güthoff, B. Hennion, C. Herzig, W. Petry, H. R. Schober, and J. Trampenau, J. Phys.: Condens. Matter 6, 6211 (1994).
${ }^{24}$ W. Petry, A. Heiming, J. Trampenau, M. Alba, C. Herzig, H. R. Schober, and G. Vogl, Phys. Rev. B 43, 10933 (1991).

${ }^{25}$ A. Heiming, W. Petry, J. Trampenau, M. Alba, C. Herzig, H. R. Schober, and G. Vogl, Phys. Rev. B 43, 10948 (1991).

${ }^{26}$ J. Trampenau, A. Heiming, W. Petry, M. Alba, C. Herzig, W. Miekeley, and H. R. Schrober, Phys. Rev. B 43, 10963 (1991).

${ }^{27}$ R. J. Birgeneau, J. Cordes, G. Dolling, and A. D. B. Woods, Phys. Rev. 136, 1359 (1964).

${ }^{28}$ S. Hautecler and W. Van Dingenen, Physica (Amsterdam) 34, 257 (1967).

${ }^{29}$ G. A. deWit and B. N. Brockhouse, J. Appl. Phys. 39, 451 (1968).

${ }^{30}$ M. Zoli, G. Santoro, V. Bortolani, A. A. Maradudin, and R. F. Wallis, Phys. Rev. B 41, 7507 (1990).

${ }^{31}$ R. J. McQueeney and R. A. Robinson, Neutron News 14, 36 (2003).

${ }^{32} \mathrm{http} / / /$ lansce.lanl.gov/lujan/instruments/Pharos/index.html

${ }^{33}$ B. T. Fultz and J. M. Howe, Transmission Electron Microscopy and Diffractometry of Materials, 2nd ed. (Springer-Verlag, Berlin, 2002), Chap. 1, Sec. 1.5.3, p. 51.

${ }^{34}$ Y. S. Touloukian, R. K. Kirby, R. E. Taylor, and P. D. Desai, Thermophysical Properties of Matter (IFI, New York, 1975), Vol. 12.

${ }^{35}$ J. R. Santisteban, L. Edwards, A. Steuwer, and P. J. Withers, J. Appl. Crystallogr. 34, 289 (2001).

${ }^{36}$ M. Born and K. Huang, Dynamical Theory of Crystal Lattices (Clarendon, Oxford, 1988).

${ }^{37}$ A. Maradudin, E. Montroll, G. Weiss, and I. Ipatova, in Solid State Physics, 2nd ed., edited by H. Ehrenreich, F. Seitz, and D. Turnbull (Academic, New York, 1971), Suppl. 3.

${ }^{38}$ R. Bechmann and R. F. S. Hearmon, Elastische Piezoelektriche, Piezooptische und Elektrooptische Konstanten von Kristallen, Landolt-Börnstein, New Series, Group III, Vol. 3 (SpringerVerlag, Berlin, 1966).

${ }^{39}$ V. P. N. Sarma and P. J. Reddy, Phys. Status Solidi A 16, 413 (1973).

${ }^{40}$ V. P. N. Sarma and P. J. Reddy, Philos. Mag. 27, 769 (1973).

${ }^{41}$ V. F. Sears, E. C. Svensson, and B. M. Powell, Can. J. Phys. 73, 726 (1995).

${ }^{42}$ E. Johnson and L. Robinson, Rev. Sci. Instrum. 60, 3447 (1989).

${ }^{43}$ G. Placzek and L. Van Hove, Phys. Rev. 93, 1207 (1954).

${ }^{44} \mathrm{G}$. L. Squires, Introduction to the Theory of Thermal Neutron Scattering (Dover, New York, 1997), Chap. 3, Sec. 10, p. 57. 